\title{
Characterising Mechanical Properties of Flowing Microcapsules Using a Deep Convolutional Neural Network
}

\author{
T. Lin ${ }^{1}$, Z. Wang ${ }^{2}$, R. X. Lu ${ }^{1}$, W. Wang ${ }^{1}$ and Y. Sui ${ }^{1, *}$ \\ 1 School of Engineering and Materials Science, Queen Mary University of London, \\ London E1 4NS, United Kingdom \\ 2 Department of Mechanical Engineering, University College London, London WC1E \\ 6BT, United Kingdom
}

Received 18 November 2020; Accepted (in revised version) 17 January 2021

\begin{abstract}
Deformable microcapsules are widely used in industries and also serve as a mechanical model of living biological cells. In this study, we develop a novel method, by integrating a deep convolutional neural network (DCNN) with high-fidelity mechanistic capsule modelling, to identify the membrane constitutive law and estimate associated parameters of a microcapsule from its steady deformed profile in a capillary tube. Compared with conventional inverse methods, the present approach is more accurate and can increase the prediction throughput rate by a few orders of magnitude. It can process capsules with large deformation in inertial flows. Furthermore, the method can predict the capsule membrane shear elasticity, area dilatation modulus and initial inflation from a single steady capsule profile. We explore the mechanism that the DCNN makes decisions by considering its feature maps, and discuss their potential implication on the development of inverse methods. The present method provides a promising tool which may enable high-throughput mechanical characterisation of microcapsules and biological cells in microfluidic flows.
\end{abstract}

AMS subject classifications: 92C10, 76Z99

Key words: Microcapsules, flow cytometry, deep convolutional neural network, high throughput, mechanical characterisation.

\section{Introduction}

A capsule consists of a liquid droplet enclosed by a thin elastic membrane [1]. Microcapsules are widely used in food, cosmetic, biomedical and pharmaceutical industries

${ }^{*}$ Corresponding author.

Email: y.sui@qmul.ac.uk (Y. Sui) 
in a vast range of applications such as controlled agent release [2], targeted drug delivery [3], and encapsulated cell culture [4]. The capsule membrane protects its internal contents and regulates mass exchange, therefore knowing its mechanical properties is crucial in the design and fabrication of capsules. Microcapsules have also been widely used as a mechanical model of living biological cells [5-7]. The capsule elasticity, inferred from overall cell deformation, can be employed as a marker-free way to quantify the cell states and properties, such as the metastatic potential and degree of differentiation [8]. It therefore has many ground-breaking biological and clinical applications $[9,10]$.

It has been very challenging to characterise the mechanical properties of microcapsules and biological cells due to their small size and fragility. A few methods have been proposed, such as the parallel plate squeezing [11], micropipette aspiration [12], optical stretching [13] and AFM measurement [14]. It is also possible to deform capsules using viscous fluid force that can be generated in shear $[15,16]$, centrifugal $[17,18]$, and extensional $[19,20]$ flows. Those methods measure the deformation of capsules or cells under well-defined stress. Their throughput rates are typically limited to 100 capsules or cells per hour, which may be low for many important applications, such as cancer diagnosis or cell sorting based on cell mechanical properties. Those applications need to measure many thousands to millions of cells in minutes to hours, and therefore require high-throughput methods capable of processing at least hundreds of cells per second [21].

In recent years, a novel hydrodynamic approach with a much higher throughput rate has been proposed. In this method, microcapsules or biological cells are flowed through a microfluidic channel with comparable cross-sectional dimension, where they are deformed by the viscous fluid force. By fitting the steady deformed capsule profile to computational or theoretical predictions, one can inversely infer the membrane mechanical law and associated parameters. This promising approach has been developed for both artificial microcapsules [22-24] and living biological cells $[6,8,9,25,26]$. The state-of-theart system can perform real-time processing of the deformation of hundreds of cells per second. However, the cell mechanical properties need to be calculated through postprocessing, due to the long processing time of inverse methods.

Recently, deep neural networks have attracted much attention in a wide range of applications, for it provides a versatile method to infer the relationship between data and their corresponding measurements. In particular, the convolutional neural networks have significant merits in processing data that comes in the form of images [27], and have led to ground-breaking applications in image processing [28], classification of cells [29], geometrical optimization of aerofoils [30], and turbulence research [31-33].

In the present study, we for the first time integrate a deep convolutional neural network (DCNN) with high fidelity mechanistic modelling to create a novel approach to identify the membrane constitutive law and estimate associated parameters of a microcapsule from its steady deformed profile when flowing in a capillary tube. Unlike conventional inverse methods which need to identify the best fit online, the present DCNN is trained offline, and its prediction process only involves a limited number of algebraic calculations. It is therefore much faster and can predict the properties of more than one 
thousand capsules per second. We also demonstrate that the present method is more accurate and has fewer restrictions.

\section{Problem statement}

We consider an initially spherical capsule of radius $a$ suspended in a Newtonian fluid flowing in a tube of radius $R$. The capsule is enclosed by a hyper-elastic membrane which is described by either the strain-softening neo-Hookean (NH) law [34] or the strainhardening Skalak's (SK) law [35]. The NH law assumes that the membrane is an infinitely thin sheet of a three-dimensional isotropic volume-incompressible material

$$
W^{N H}=\frac{1}{2} G_{s}\left(I_{1}-1+\frac{1}{I_{2}+1}\right),
$$

where $W$ is the strain energy density per unit undeformed surface area, $G_{s}$ is the membrane shear elasticity modulus, $I_{1}$ and $I_{2}$ are the first and second strain invariants of the surface deformation. The SK law is designed for biological cells and assumes a strain energy function

$$
W^{S K}=\frac{1}{4} G_{S}\left(I_{1}^{2}+2 I_{1}-2 I_{2}\right)+\frac{1}{4} C G_{s} I_{2}^{2}
$$

where the dimensionless parameter $C$ measures the resistance to membrane area dilatation. It indicates the strength of the strain-hardening nature of the SK membrane. In the limitation of small capsule deformation, the membrane area dilatation modulus $K_{s}$ is related to the shear elasticity by $K_{s}=(1+2 C) G_{s}$. For biological membranes, $C$ is usually much larger than unity, because of their quasi area incompressibility. However, for artificial capsules, the SK law has been found to fit experimental data well when $C$ is of order 1 [11]. In the present study, we set $C=1$ in most simulations unless otherwise specified. The bending resistance of the capsule membrane is modelled using Helfrich's formulation [36,37], with a small bending rigidity to prevent the formation of membrane wrinkles. The present model is therefore valid mainly for capsules with a thin membrane, where the membrane bending has negligible effect on global deformation of the capsules [38]. The fluids inside and outside the capsule are assumed to have identical dynamic viscosity and density. Note that the ratio of dynamic viscosity between the fluids inside and outside the capsule has no effect on the steady profile that the capsule reaches in tube flows [39]. This is because the internal fluid is largely in solid translation after the capsule membrane has achieved a steady shape.

Under the viscous force of the tube flow, the capsule deforms to a steady profile depending on the following dimensionless parameters:

- the flow Reynolds number $\operatorname{Re}=\frac{\rho U(2 R)}{\mu}$, where $\rho$ and $\mu$ represent the fluid density and dynamic viscosity, respectively, and $U$ is the mean velocity of the tube flow;

- the size ratio $\beta=\frac{a}{R}$, which compares the size of the capsule to that of the tube; 
- the capillary number $C a=\frac{\mu U}{G_{s}}$, which represents the ratio of viscous and elastic forces;

- the pre-inflation ratio $\alpha=\frac{a}{a_{0}}-1$, which relates the initial inflated radius $a$ to the radius $a_{0}$ of the capsule at unstressed configuration.

\section{Computational method}

The present method predicts the membrane constitutive law and associated parameters of a capsule from its steady deformed profile in tube flow. The method consists of two parts: the first part is a mechanical approach to simulate the deformation of a capsule in tube flow to obtain its steady shape. The present simulations have been based on an immersed-boundary lattice Boltzmann method, which has been validated extensively for deformation of capsules in shear and tube/channel flows [40-46]. Details of the method can be found in pertinent literature and are not described here. The simulations results, mainly the steady deformed capsule profile, the corresponding membrane constitutive law and associated parameters, are then used to train a DCNN, which represents the second part of the present method. The present DCNN is detailed below.

\subsection{Structure of the DCNN}

The present DCNN belongs to supervised learning. It learns functions mapping the inputs, which are binary images of deformed capsules, to the measurements, that are membrane constitutive laws and associated parameters. The DCNN is developed using the open-source software Tensorflow [47] with Keras Application Programming Interface (API) [48]. Its architecture is shown in Fig. 1. There are four convolutional blocks, within

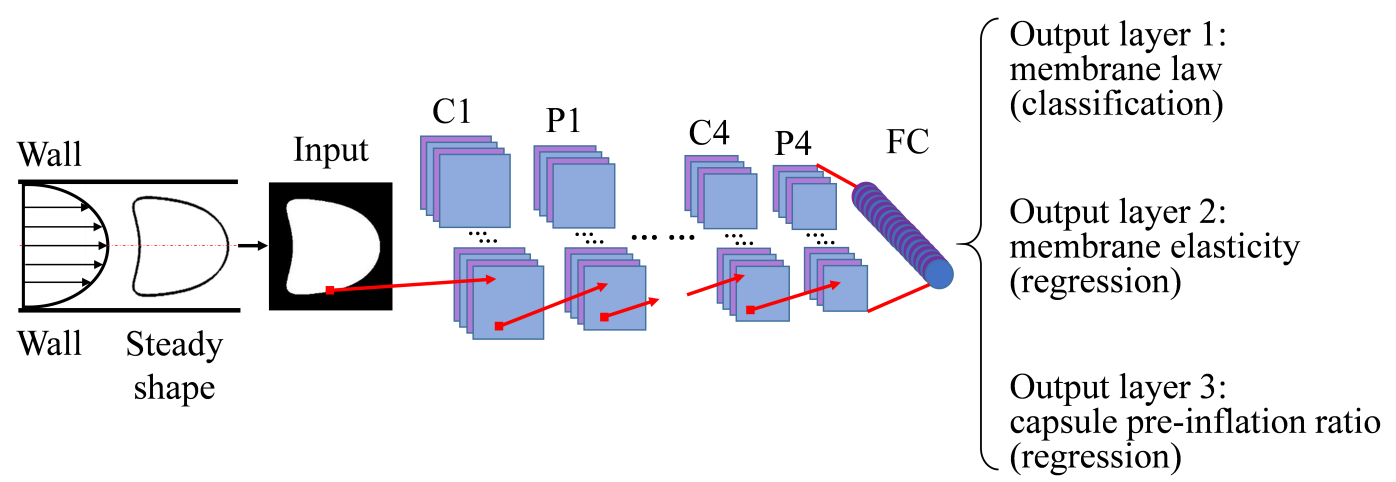

Figure 1: Architecture of the present convolutional neural network which predicts membrane mechanical properties of a microcapsule from its steady deformed profile when flowing in a capillary tube. Details of the convolutional $(C)$, pooling $(P)$, fully connected $(F C)$ and output layers are elaborated in the text. 


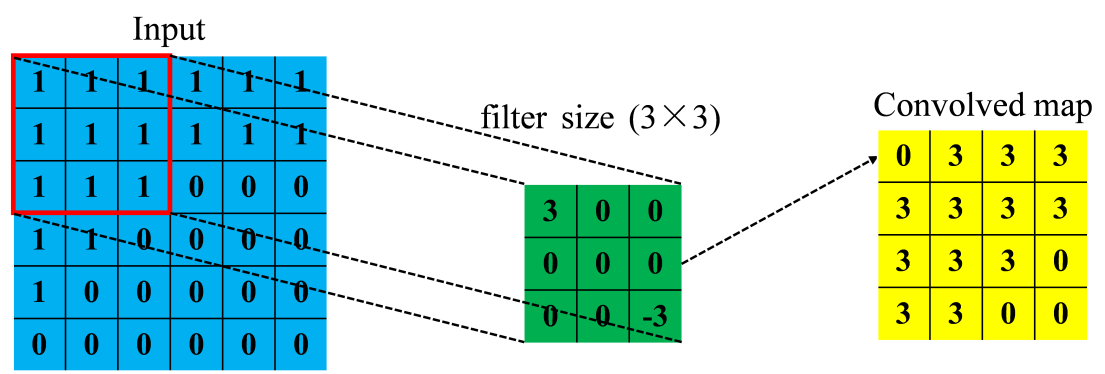

Figure 2: Convolution operation in the convolutional layer.

each a convolutional and a pooling layers are in sequence. These are followed by a fullyconnected layer and a few output layers. Details of the different layers are elaborated below.

The convolutional layer consists of a number of filters, which are smaller in spatial dimensions compared with the input image. During the convolution operation between each filter and the input image, the filter slides through the entire image and the filter weights make an elementwise scalar product with each small region of the image (see Fig. 2), following [30]:

$$
\boldsymbol{x}_{i j}=(\boldsymbol{I} * \boldsymbol{w})_{i j}=\sum_{m=0}^{l_{1}-1} \sum_{n=0}^{l_{2}-1} \boldsymbol{w}_{m, n} \cdot \boldsymbol{I}_{i+m, j+n},
$$

where $I$ represents the input image which has $l$ and $h$ pixels along directions of the length and height, respectively. The term $w$ is the weights of the filter with a size of $l_{1} \times l_{2}$. The indexes $i, j$ of the scalar product range from 0 to $l-l_{1}$ and $h-l_{2}$, respectively. The output of the convolution operation follows:

$$
\boldsymbol{o}_{i j}=\sigma\left(\boldsymbol{x}_{i j}+\boldsymbol{b}\right),
$$

where $\boldsymbol{b}$ is a bias term which is independent of $i$ and $j$, and $\sigma$ is a nonlinear activation function, which we have used the rectified linear unit (ReLU) function:

$$
\sigma(z)=\max (0, z)
$$

In Eq. (3.3), the output of the ReLU function is 0 when $z<0$. The ReLU function has been shown to have superior ability to train DCNNs faster [49].

The pooling layer usually follows the convolutional layer and operates on the output of the convolutional layer to reduce its dimension. In the present study, we have employed the maximum pooling operation which gives the maximum of the numbers in the pooling kernel (see Fig. 3). The shared fully connected layer follows the pooling layer and contains neurons that have full connections to the pooling layer. 


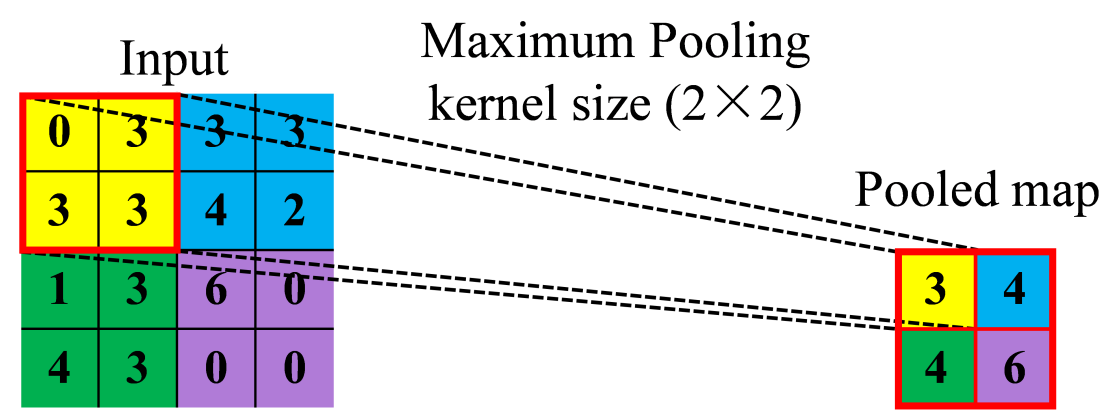

Figure 3: Maximum pooling operation in the pooling layer.

The present DCNN is ended with a few output layers, which can conduct multiple classification and regression tasks. A classification output layer predicts the class (or type, such as NH or SK) of the membrane constitutive law of a capsule. This is done through choosing the membrane law type that has the highest probability predicted by the DCNN from the input capsule image. The regression output layers directly predict the values of capsule membrane shear elasticity $G_{s}$ and the pre-inflation ratio $\alpha$ etc. Details of the classification and regression tasks are provided in the following Section 3.2.

\subsection{Training of the DCNN}

The DCNN needs to be trained on a set of examples. In the present study, each example consists of a binary image of a deformed capsule, and multiple corresponding measurements such as the membrane constitutive law and associated parameters. The training examples are obtained from numerical simulations using an immersed-boundary lattice Boltzmann method.

During training, the internal parameters of the DCNN are adjusted to minimize a loss function that describes how close the predictions are to the ground truth. The total loss consists of the losses from classification and regression tasks. For classification we use the cross-entropy loss function following [50]:

$$
L_{i}^{c l s}=-\left(y_{i}^{c l s} \log \left(p_{i}\right)+\left(1-y_{i}^{c l s}\right) \log \left(1-p_{i}\right)\right)
$$

where $p_{i}$ represents the probability (predicted by the DCNN) that the capsule follows the SK law, and $y_{i}^{c l s}$ is the ground-truth label of the input image $\left(y_{i}^{c l s}=0\right.$ represents SK law, $y_{i}^{c l s}=1$ represents NH law).

We use the square loss function for regression tasks:

$$
L_{i}^{r e g}=\left\|y_{i}^{r e g}-y_{i}\right\|^{2},
$$


where $y_{i}$ represents the predicted quantity of $G_{s}$ or $\alpha$ by the present DCNN and $y_{i}^{r e g}$ is the corresponding ground-truth. The total loss function follows:

$$
L_{\text {total }}=\frac{1}{N} \sum_{i=1}^{N}\left(L_{i}^{c l s}+L_{i}^{r e g}\right)
$$

where $N$ is the number of training samples.

To optimize the trainable internal parameters, we obtain the gradients of the loss function $L_{\text {total }}$ with respect to these parameters using a backpropagation algorithm [51], and then an optimizer using a stochastic gradient descent algorithm called ADAM [52] is applied to update the values of the parameters. Mini-batch mode training is adapted, and exposing all training samples to the DCNN once is called an epoch. At the end of each epoch, the DCNN is validated with a small portion (i.e., 10\% in the present study) of the training samples that have not been used during training. With the process iterates the total loss decreases and converges towards small values (typically at the order of $10^{-3}$ ). To avoid overfitting, a batch-normalization [53] and a dropout regularization [54] have been employed in the present DCNN. The training process is terminated with predefined early stopping criteria, when the total loss of validation shows no further improvement over several iterations even after reducing the learning rate.

After training and validation, the DCNN is used to predict the membrane law type, membrane shear elasticity $G_{s}$ and capsule pre-inflation ratio $\alpha$ from the steady deformed image of a capsule.

\subsection{Choices of hyperparameters}

The performance of the present DCNN is affected by its certain hyperparameters. In the present study we conduct extensive tests to identify the optimum set of hyperparameters that leads to the smallest converged total loss (defined in Eq. (3.6)) during training and validation. Our test results (not shown) suggest that the following choices of hyperparameters lead to the optimum prediction accuracy of the present DCNN: the network has four convolutional blocks, and the number of filters in each of the four convolutional layers is $16,32,64$, and 128 , respectively. The size of filter for convolutional layers is $3 \times 3$ with a stride of one and the same padding operation. The max-pooling kernel size is $2 \times$ 2 with a stride of two and the same padding operation. There is one fully connected layer with 256 neurons. During training, the size of the mini-batch is chosen to be 32. An initial learning rate of 0.001 is used with a learning rate scheduler, which reduces the learning rate in steps when the total loss of validation shows no improvement over epochs. In the dropout regularization to avoid overfitting, the dropout rate is set as 0.7. 


\section{Results and discussion}

\subsection{Tests against simulation results}

We first test the performance of the present DCNN by predicting the membrane constitutive law and capillary number $\mathrm{Ca}$ from the cross-sectional profile of a steady deformed capsule. The membrane shear elasticity $G_{s}$ is related to $C a$ by $G_{s}=\mu U / C a$. All the samples are obtained from numerical simulations based on an immersed-boundary lattice Boltzmann method. The training samples consist of 200 sets of binary images (with 300 pixels in both directions), equally covering $\mathrm{NH}$ and SK laws, two capsule sizes $\beta=0.77$ and 0.89 , with $\mathrm{Ca}$ ranging from 0.001 to 0.055 . The flow is in Stokes regime and the capsule pre-inflation ratio is fixed at $3 \%$. In this parameter space, there are high-quality experimental results available [39], and a capsule can achieve moderate to large deformation. The testing samples are within the same parameter regime, and are not used during training and validation. We find for all 20 testing samples, the present DCNN has correctly predicted the types of membrane constitutive laws, and this is shown in Fig. 4(a). The predicted $\mathrm{Ca}$ are compared with the corresponding ground truth in Figs. 4(b) and (c), where very good agreements can be observed. The mean absolute percentage error (MAPE) of the predicted $\mathrm{Ca}$ from the corresponding ground truth calculated from the entire testing samples is $2.1 \%$, which suggests excellent prediction accuracy. Note that MAPE of a parameter $A$ is defined as:

$$
\operatorname{MAPE}(A)=\frac{1}{M} \sum_{i=1}^{M}\left|\frac{A_{\mathrm{pre}, i}-A_{\mathrm{gt}, i}}{A_{\mathrm{gt}, i}}\right|,
$$

where $A_{\mathrm{pre}, i}$ and $A_{\mathrm{gt}, i}$ are the predicted and ground-truth values of the $i^{\text {th }}$ testing sample; $M$ is the total number of testing samples. From Figs. $4(\mathrm{~b})$ and (c), it is also seen that the steady profiles of capsules with $\mathrm{NH}$ and SK membranes are very similar at low $\mathrm{Ca}$ in small deformation regime, which is consistent with previous studies [55]; however, with $\mathrm{Ca}$ increasing, a capsule with an $\mathrm{NH}$ membrane undergoes considerably larger deformation compared with a capsule with an SK membrane at the same $\mathrm{Ca}$, due to the strain-softening nature of the NH membrane. From Fig. 4(d), we can find that the MAPE decreases with $\mathrm{Ca}$, which suggests the DCNN generally performs better when the capsule deformation is large.

It should be noted that the present mechanical models for flows and capsules can readily account for inertial effect [45], which is relevant to inertial microfluidics where the average flow speed can reach meters per second and the flow Reynolds number achieves the order of $100[8,26]$. We conduct studies similar to those in Fig. 4 and consider a capsule with $\beta=0.77$ at a much higher $\operatorname{Re}=40$ in the inertial flow regime. In practical experiments, for a microcapsule with a radius of $50 \mu \mathrm{m}$ suspended in water at room temperature, a flow Reynolds number of $R e=40$ can be achieved with an average flow speed of $0.31 \mathrm{~m} / \mathrm{s}$. This corresponds to a pressure drop of $5.89 \mathrm{kpa}$ per centimeter tube length in a tube with a radius of $65 \mu \mathrm{m}(\beta=0.77)$. We find that the inertial effect increases capsule deformation at 

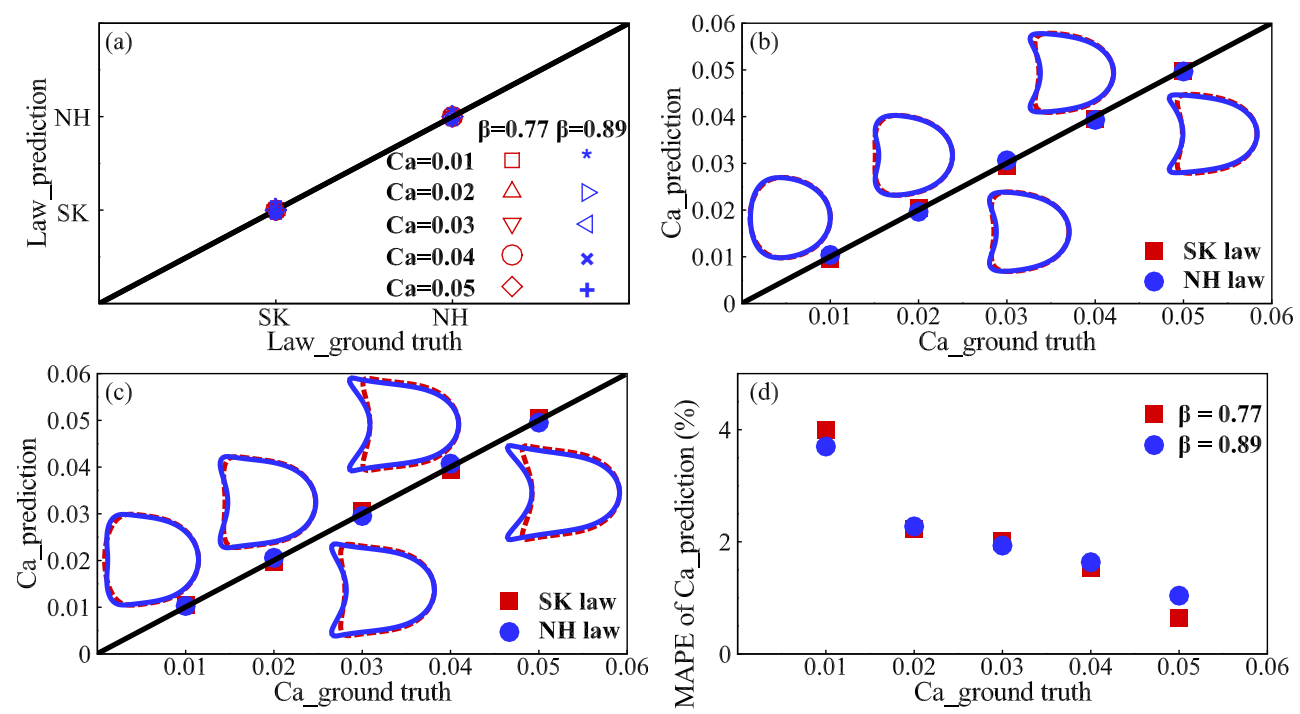

Figure 4: Comparisons of predicted (a) membrane constitutive law and (b)-(c) capillary number $\mathrm{Ca}$ with the corresponding ground truth. The membrane shear elasticity $G_{s}$ is related to $C a$ by $G_{s}=\mu U / C a$. The solid lines are used as guides for the eyes representing perfect agreement. Parameters are $\operatorname{Re}=0.2, \alpha=3 \%, \beta=(b) 0.77$; (c) 0.89. Insets in (b)-(c) are cross-sectional profiles of capsules with NH (blue solid) and SK (red dashed) membrane at the corresponding $\mathrm{Ca}$, which show extents of deformation. (d) MAPE of predicted $\mathrm{Ca}$ from the corresponding ground truth for $\beta=0.77$ and 0.89 , respectively.

the same value of $C a$. However, the prediction accuracy of the present DCNN is similar to that in Fig. 4.

The present DCNN can be conveniently extended to multiple classification and regression tasks, by adding more parallel output layers. Here we demonstrate its extension to the prediction of the capsule pre-inflation ratio $\alpha$, besides the membrane constitutive law and $C a$. In experiments, capsules with polymeric membranes in microchannel flows have been found to be slightly inflated due to the osmotic effect $[22,39]$. Here we consider a capsule with $\beta=0.77$. All the samples are obtained from numerical simulations. The training samples have been extended from those used in Fig. 4, to include additional 400 cross-sectional images of capsules with $\alpha=1 \%, 2 \%, 4 \%$ and $5 \%$, covering both $\mathrm{NH}$ and SK laws. An increase of the training sample size has resulted in a longer training time, which is about 5 hours using a Tesla GPU (V100-16GB, 1.38GHz). The testing samples cover both membrane laws, five values of $\alpha$, and five values of $C a$, and consist of $2 \times 5 \times 5=50$ images. Again the present DCNN predicts the membrane constitutive laws of all 50 testing samples correctly; however, to save space this is not shown in a figure. Figs. 5(a) and (b) compare the predicted capsule pre-inflation ratio and capillary number with their corresponding ground truth for the 50 testing samples. The mean absolute percentage error of $\alpha$ and $C a$ from their ground truth are $4.5 \%$ and $2.6 \%$, respectively.

We also extend the DCNN used in Fig. 4 to predict the membrane dilatation param- 

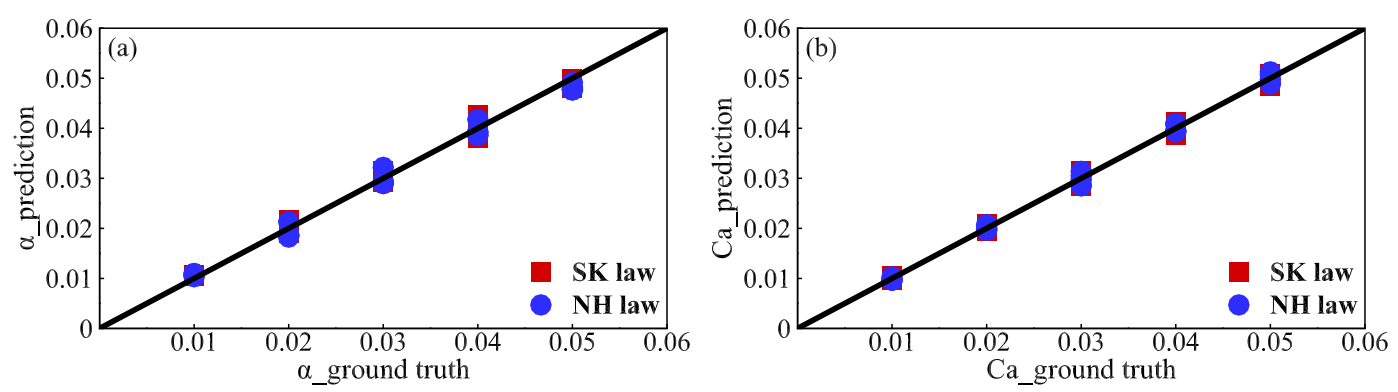

Figure 5: Comparison of predicted capsule pre-inflation ratio $\alpha$ and capillary number $\mathrm{C} a$ with the corresponding ground truth. The solid lines are used as guides for the eyes representing perfect agreement. Parameters are $\mathrm{Re}=0.2, \beta=0.77$.
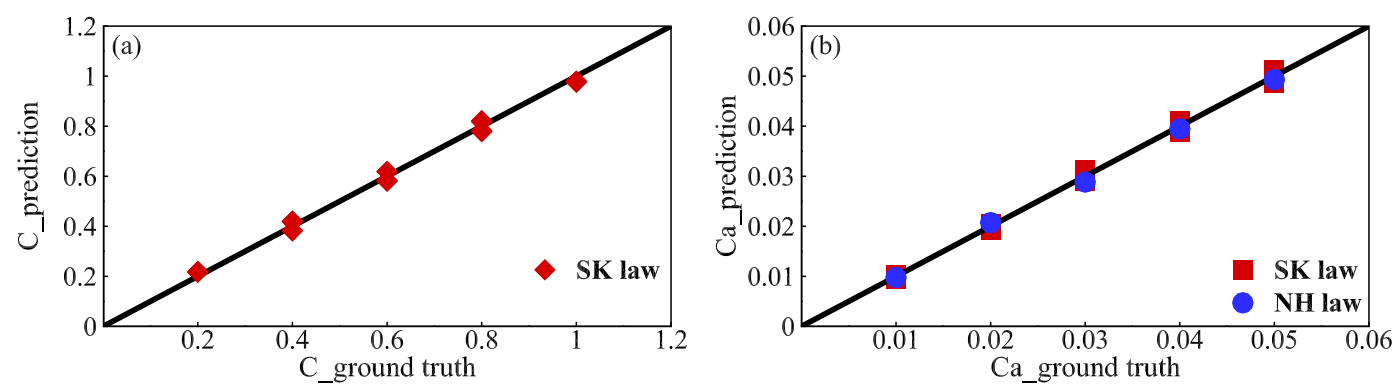

Figure 6: Comparison of predicted membrane dilatation parameter $C$ (associated with the SK membrane) and capillary number $\mathrm{Ca}$ with the corresponding ground truth. The solid lines are used as guides for the eyes representing perfect agreement. Parameters are $\operatorname{Re}=0.2, \alpha=3 \%, \beta=0.77$.

eter $C$ of capsules with an SK membrane. The parameter is related to the membrane area dilatation modulus by $K_{s}=(2 C+1) G_{s}$. Note that for such capsules, both the shear elasticity and the membrane dilatation parameter affect the capsule deformation. In previous studies multiple experiments are commonly needed to disentangle their individual contribution and infer their values. Here we consider a capsule with $\beta=0.77$. The training samples have been extended from those used in Fig. 4, to include additional 200 cross-sectional images of capsules enclosed by an SK membrane with the membrane area dilatation parameter ranging from 0.2 to 1 . The testing samples consist of 30 crosssectional images of capsules that have not been used during the training phase. These include 25 images for capsules with an SK membrane covering five values of $C$ and five values of $\mathrm{C} a$, and 5 images for capsules with an $\mathrm{NH}$ membrane covering five values of $\mathrm{Ca}$. The present DCNN predicts the membrane constitutive laws of all 30 testing samples correctly. Fig. 6(a) shows the comparison between the predicted $C$ with the corresponding ground truth for the 25 testing images of capsules with an SK membrane. Fig. 6(b) compares the predicted capillary number and the corresponding ground truth for all the 30 testing samples covering both membrane laws. Good agreements can be observed 
in both figures. The MAPE of $C$ and $C a$ from their ground truth are $4.3 \%$ and $2.7 \%$, respectively. The present results therefore suggest that it may be possible to reasonably accurately predict the membrane constitutive law and estimate multiple associated parameters from the steady profile of a capsule with moderate/large deformation in tube flows. We will further discuss this point in Section 4.3.

\subsection{Prediction using the footprint profile of a capsule}

The present method can conveniently process capsules with large deformation, where the rear region of capsule forms a concave parachute shape (see the photo on the left of Fig. 7(a)). For a capsule under large deformation, it becomes difficult to obtain the cross-sectional profile because part of it hides in the shadow. In previous studies, it was necessary to manually erase the concave region to obtain the cross-sectional shape (see the photo in the upper row of Fig. 7(a)) [39], which may cost considerable processing time.

To solve this problem, we test if the present DCNN can make accurate predictions from the footprint shape of a capsule (see the image in the lower row of Fig. 7(a)). The
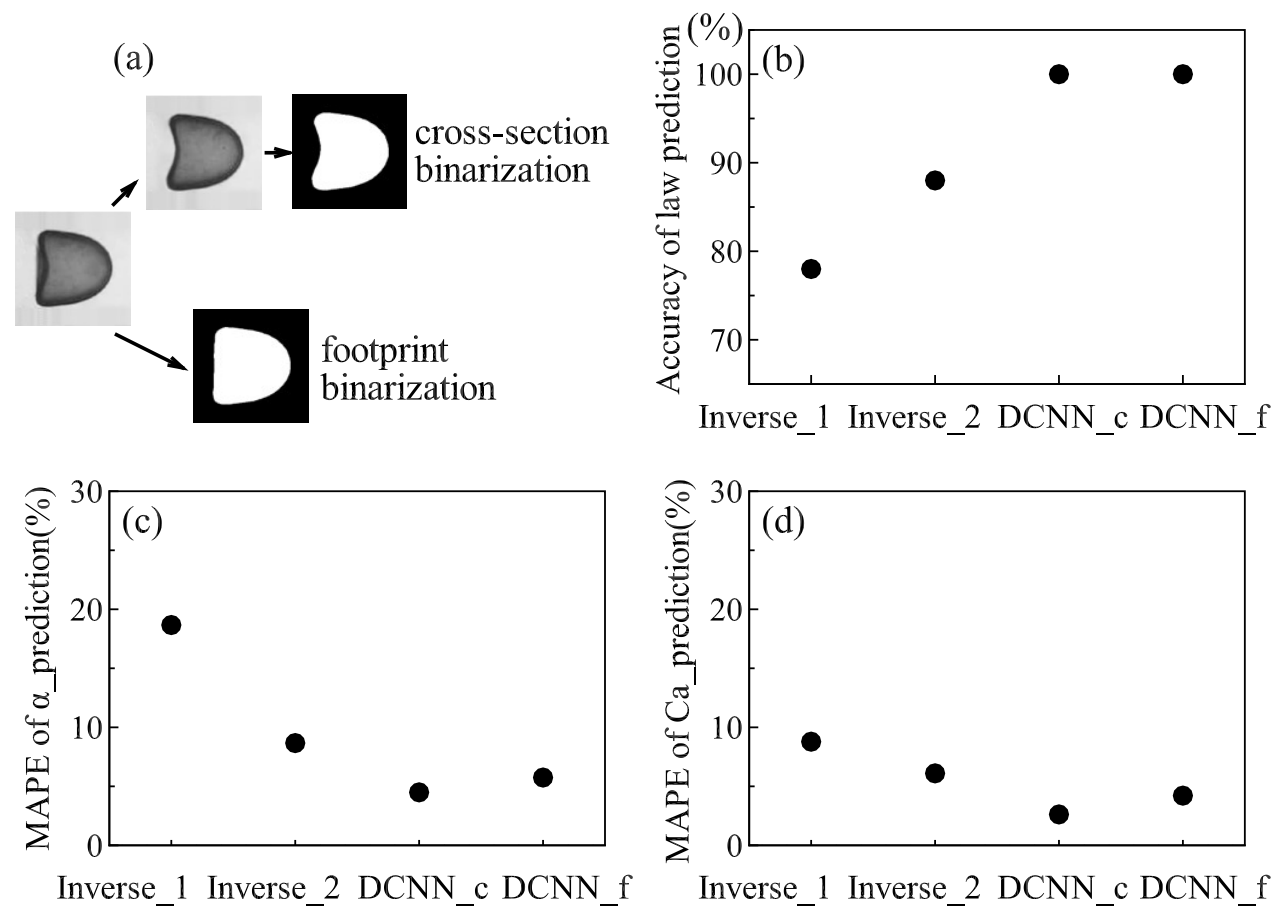

Figure 7: (a) Illustration of cross-section binarization and footprint binarization. Photos are taken from [39]. Comparisons of (b) prediction accuracy of membrane constitutive laws, (c) MAPE of predicted $\alpha$, (d) MAPE of predicted $\mathrm{Ca}$ from different methods with the same training and testing samples of Fig. 3. 
training and testing samples, using footprint profiles, are prepared from the same cases employed in Fig. 5. The accuracy of the new method, labelled as DCNN_f, is shown in Figs. 7(b)-(d). From Fig. 7(b) it is seen that DCNN_f has predicted the laws of all 50 testing samples correctly. The mean absolute percentage errors of $\alpha$ and $C a$ from the DCNN_f are $5.7 \%$ and $4.2 \%$, respectively. These are only slightly higher than those from the DCNN using the cross-sectional shapes of capsules (labelled as DCNN_c), which contain more geometrical information of the rear region of the capsules. Note that when $C a \leq 0.01$, the capsule deformation is small and its footprint profile closely resembles the cross-sectional shape. With the ten testing samples at $C a=0.01$ not included, the MAPEs of $\alpha$ and $C a$ of the DCNN_f are $5.3 \%$ and $3.9 \%$, respectively, compared with the corresponding values of $4.3 \%$ and $2.2 \%$ of the DCNN_c.

\subsection{Comparison with inverse methods}

We compare the prediction accuracy of the present methods with two types of conventional inverse methods. The first type compares simple unique features of a deformed capsule measured in the experiment, such as the total length and parachute depth along the flow direction, with those from a data bank that have been obtained by numerical simulations. The membrane constitutive law and associated parameters are inferred from the simulation result that gives the best fit [24].

The second type of inverse method is similar to the first type in that it also infers properties from the best fit, however, the second type of method compares the entire profile of a deformed capsule in experiment (i.e., $C_{e}$ ) with those obtained from numerical simulations $C_{s}$ [6]. The difference of two profiles can be quantified with a parameter, such as the mean Hausdorff distance (MHD) [56]. The minimum MHD indicates the best fit. To explain the MHD, let's considers a set of $m$ points, $R=\left\{r_{1}, r_{2}, r_{3}, \cdots, r_{m}\right\}$ from $C_{e}$, and another set of $n$ points, $T=\left\{t_{1}, t_{2}, t_{3}, \cdots, t_{n}\right\}$ from $C_{s}$. Assuming that the two sets of points have the same centre of mass, the $\operatorname{MHD} \bar{h}(R, T)$ is defined as:

$$
\bar{h}(R, T)=\frac{1}{m} \sum_{r \in R} \min _{t \in T}[d(r, t)],
$$

where $d(r, t)$ is the distance from any point in $R$ to any point in $T$.

Figs. 7(b)-(d) show the accuracy of the two inverse methods and the present approaches in predicting membrane constitutive laws, pre-inflation ratio and capillary number. It should be noted that the same training and testing samples used in Fig. 5 have been employed here for all four methods. In both inverse methods, the training samples of Fig. 5 are used as the data bank, which provides images that a testing image can be compared with. It is not surprising to see from Fig. 7 that the present method is the most accurate. We find that the first inverse method does not perform well when capsule deformation is small. There the NH and SK laws give similar predictions (see insets in Fig. 4 at low $\mathrm{Ca}$ ) and the capsule total length is not sensitive to $\alpha$ and $C a$. The present DCNN learns features from the entire deformed profile of a capsule which may have contained 
Table 1: Throughput rates of different methods.

\begin{tabular}{||l|c|c|c||}
\hline Throughput rate / Method & Inverse method 1 & Inverse method 2 & DCNN \\
Image processing (image / s) & 714 & 714 & 2225 \\
Prediction (image / s) & 208 & 0.03 & 1400 \\
\hline
\end{tabular}

unique individual contribution from the membrane constitutive law and its associated parameters. The present method is therefore closer to the second inverse method. In the second inverse method, the cross-sectional profile of a capsule membrane has been discretized into 128 elements. In the present DCNN, both the training and testing images have 300 pixels in both directions. The cross-sectional and footprint profiles of a capsule membrane are covered by more than 500 pixels, which are considerably more than the number of elements used in the second inverse method and have therefore resulted in higher prediction accuracy. We further discuss the accuracy of the present DCNN in the following Section 4.4.

We also compare the throughput rates of the two inverse methods and the present DCNN in Table 1. The whole prediction process consists of two steps. The first step is image processing where the original image of a deformed capsule is converted into a form that can be used as an input by a prediction method. In the second step a prediction method calculates the capsule membrane constitutive law and associated parameter values. For the two types of inverse methods, image processing mainly involves edge detection and segmentation of the cross-sectional profile of a capsule membrane into 128 equal-sized elements. This can be done conveniently using the standard subroutines in Matlab and about 714 images can be processed within a single second using a desktop personal computer (Intel Core i7, 4GHz). For the present DCNN, image processing is mainly a binarization process where a capsule image is represented by $300 \times 300$ pixels with value 1 inside the capsule and 0 outside (see Fig. 7(a)). A matlab subroutine can process 2225 images per second with the same desktop computer.

Regarding the prediction throughput rate, the first inverse method is much more efficient than the second one since it only considers the total length and parachute depth of a capsule. Similar to the second inverse method, the present DCNN considers global deformation, however, it is quite interesting to see from Table 1 that the present DCNN is faster than the second type of inverse method by four orders of magnitude. The much higher throughput rate of the present method is mainly due to two reasons. First, unlike a conventional inverse method which needs to compare a test sample with a large number of samples stored in a data bank online to find the best fit, the present DCNN has completed training off-line before making a prediction, and the prediction process only involves a limited number of algebraic calculations. Second, computations of DCNN are GPU based and utilize parallel computing, which is the case for the present prediction (GPU model: Tesla V100-16GB, 1.38GHz).

We compare the computational resources required by the two inverse methods and the DCNN in Table 2. For tests of this section, 500 samples have been employed either 
Table 2: Computational resources required by different methods.

\begin{tabular}{||l|c|c|c|}
\hline Computational resources / Method & Inverse method 1 & Inverse method 2 & DCNN \\
CPU hours for samples generation & 474,000 & 474,000 & 474,000 \\
Size of data (megabytes) & 0.03 & 1.97 & 42.93 \\
Size of memory in prediction (megabytes) & 0.19 & 4.23 & 316.33 \\
\hline
\end{tabular}

for training of the DCNN or as a data bank in the inverse methods. It takes 474,000 CPU hours to run the mechanical model and generate the data with a high-performance computing cluster (CPU: Intel E5-2650 2.2GHz). For prediction, the data of the first and second inverse methods are capsules lengths, and 2D coordinates of the membrane points, respectively; while the DCNN requires $2 \mathrm{D}$ binary images of deformed capsules. The complexity of the methods increases significantly, from the first inverse method to the DCNN. Therefore both the data size and the required memory increase exponentially. It is worth mentioning that inverse methods necessarily have many conditional statements, which can significantly degrade the performance of parallel computing.

Note that both the DCNN and inverse methods are algorithms to predict capsule membrane mechanical properties. They are independent of the mechanical model that predicts the deformed profile of the capsule in the tube. The present method can deal with capsules undergoing large deformation in inertial flows, mainly due to the versatility of the present mechanical model. Cross-sectional or footprint profiles of capsules in inertial flows can also be processed by inverse methods. However, we expect that the accuracy and throughput rate will be similar to those presented in this section and be lower than those of the DCNN.

\subsection{Feature maps of the DCNN}

It is very interesting to investigate what features of the capsule profiles have been learned by the DCNN to make the predictions of membrane law type and parameter values. Identifying those features not only helps to open the black box of the DCNN, but also provides useful information for the development of physics-based inverse methods to predict capsule properties from its deformation.

Before visualizing features learned by the DCNN, we consider the effects of membrane constitutive law, capillary number $C a$ and pre-inflation ratio $\alpha$ on the steady deformed profiles of capsules. A few examples are shown in Fig. 8(a). In the figure in each comparison, we have only varied a single parameter. From the comparisons, it can be clearly seen that the steady profiles of capsules are most affected by the membrane constitutive law and parameters in two regions: the rear corners (region A) and the parachute trough (region B).

Next, we consider the feature maps of the present DCNN. In the DCNN, both the convolutional and pooling layers use sliding filters. The outputs are known as feature maps. They present not only the strength of the activations (or responses), but also their 
(a)

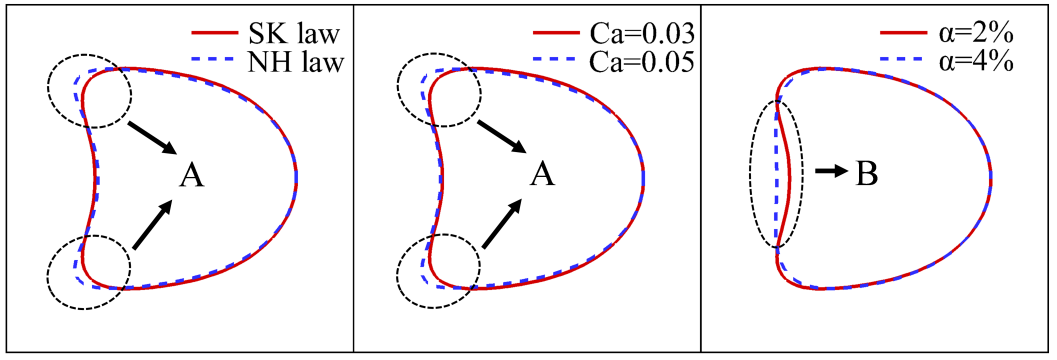

(b)

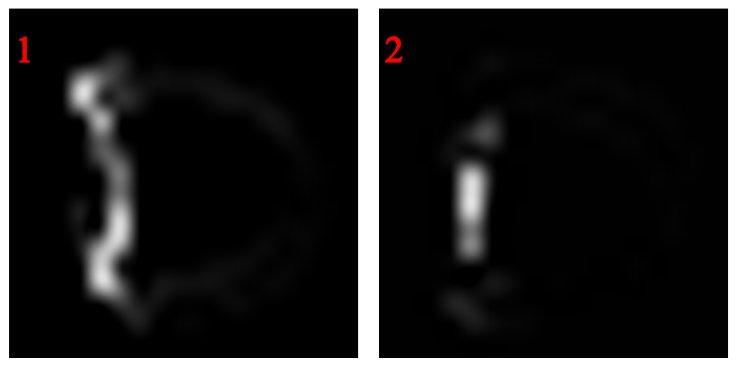

Figure 8: (a) Comparisons of the steady deformed cross-sectional profiles of capsules with different membrane constitutive law (left, $\mathrm{C} a=0.03, \alpha=2 \%$ ), capillary number $\mathrm{Ca}$ (middle, SK membrane with $\alpha=2 \%$ ) and preinflation ratio $\alpha$ (right, SK membrane with $\mathrm{Ca}=0.03$ ). In the figures, regions $\mathrm{A}, \mathrm{B}$ highlight the regions of the capsule profiles where the membrane law and associated parameters have the most significant effects. (b) Feature maps of some P4 filters corresponding to the steady cross-sectional profile of a capsule with the SK law at $C a=0.03$ and $\alpha=2 \%$. Bright color denotes high activation while dark color denotes low activation.

spatial locations. Since the features learned by the high-level layer have included those learned by layers of lower levels, here we only focus on the feature maps of some filters of the level 4 pooling layer (i.e., P4 in Fig. 1).

Fig. 8(b) presents the feature maps of some P4 filters corresponding to the steady deformed cross-sectional profile of a capsule with the SK law at $C a=0.03$ and $\alpha=2 \%$. In the feature maps, the strength of activation is indicated by the local brightness. The feature maps indicate regions of the capsule profile that the DCNN model uses to perform predictions. When comparing the feature maps with Fig. 8(a), it is quite interesting to find that the high response regions of the feature maps are corresponding to regions of the capsule profile where the capsule membrane law and associated parameters have the most significant effect. The overlaps suggest that the DCNN has successfully learned the locations of the capsule profile that are the most sensitive to the membrane laws and parameters. It is therefore not surprising that the present DCNN can perform accurate predictions, even with capsules under small deformation.

The feature maps of the present DCNN also suggest that particular attention should be paid to the parameter-sensitive regions when building high-accuracy inverse methods to predict the mechanical properties of capsules from their steady deformed profiles in tube flow. 


\subsection{Tests against experiments}

Finally, we use the same DCNN of Fig. 5 to predict the membrane constitutive law and shear elasticity of bioartificial capsules with polymeric membrane. In the experiments [39], capsules with different sizes were flowed through a tube at different flow strength in Stokes flow regime. The tube is long enough for a capsule to deform to a steady profile, which was captured by a CCD camera. The capsule membrane elasticity was characterised in separated experiments by parallel plates compression [57]. In the present study, we have concentrated on capsules with $\beta=0.77$ and 0.89 , and have used the binarized steady deformed profiles of capsules (taken from [39]) as inputs in our DCNN, which then predicts membrane constitutive law, pre-inflation ratio and membrane shear elasticity.

The present DCNN has predicted the membrane constitutive law as the SK law for all cases. The predicted capsule pre-inflation ratio $\alpha$ ranges from $2 \%$ to $4 \%$ with a mean
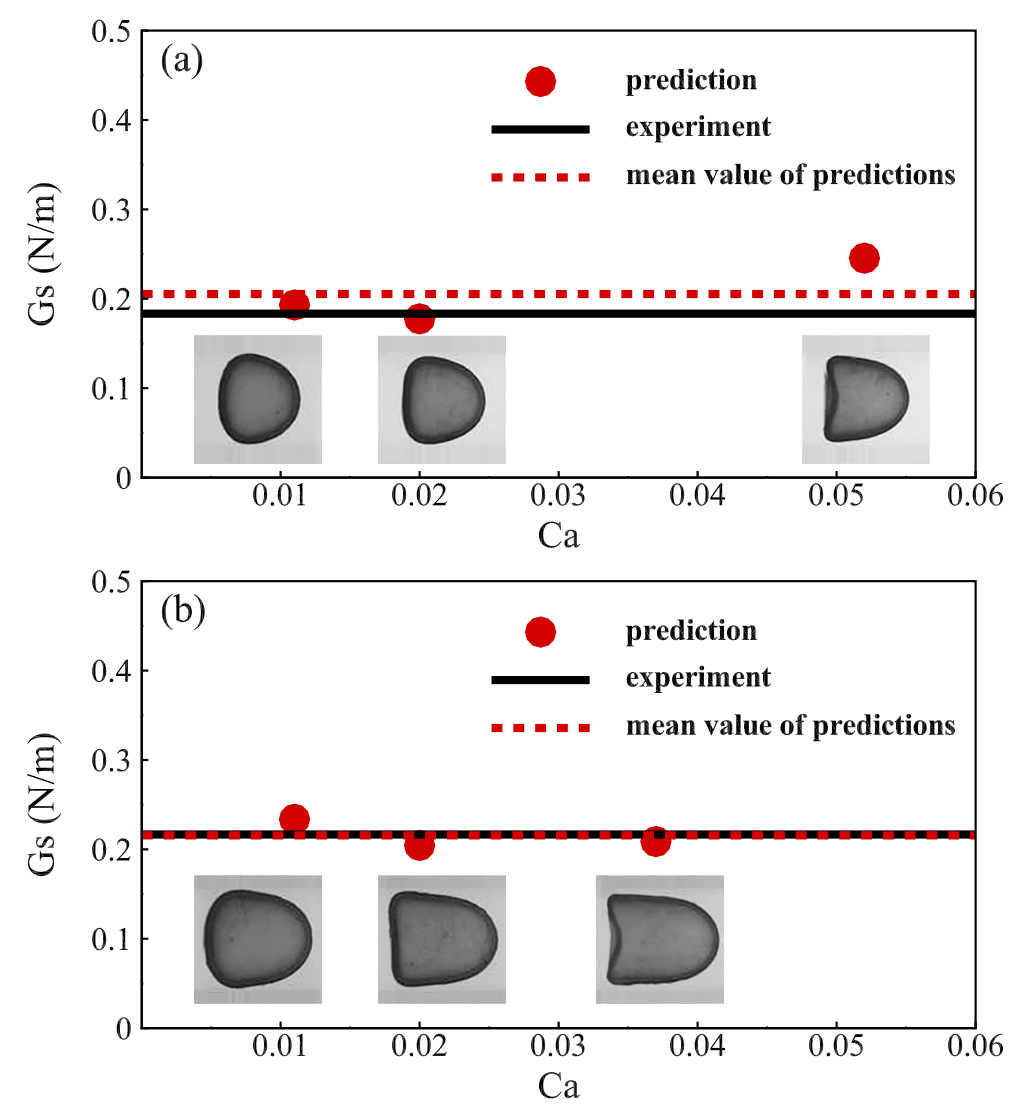

Figure 9: Comparison of predicted membrane shear elasticity with those reported in experiments [39]. The capsule is flowed through a tube at different flow strength, leading to different values of $C a . \beta=(a) 0.77$; (b) 0.89. Photo insets are images of steady deformed capsules at the corresponding flow strength from [39]. 
(a)

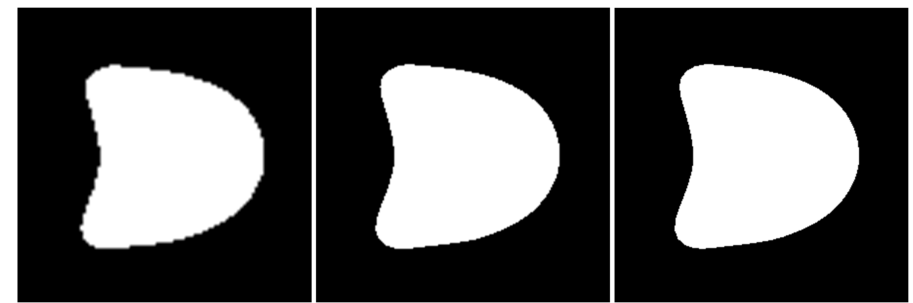

(b)

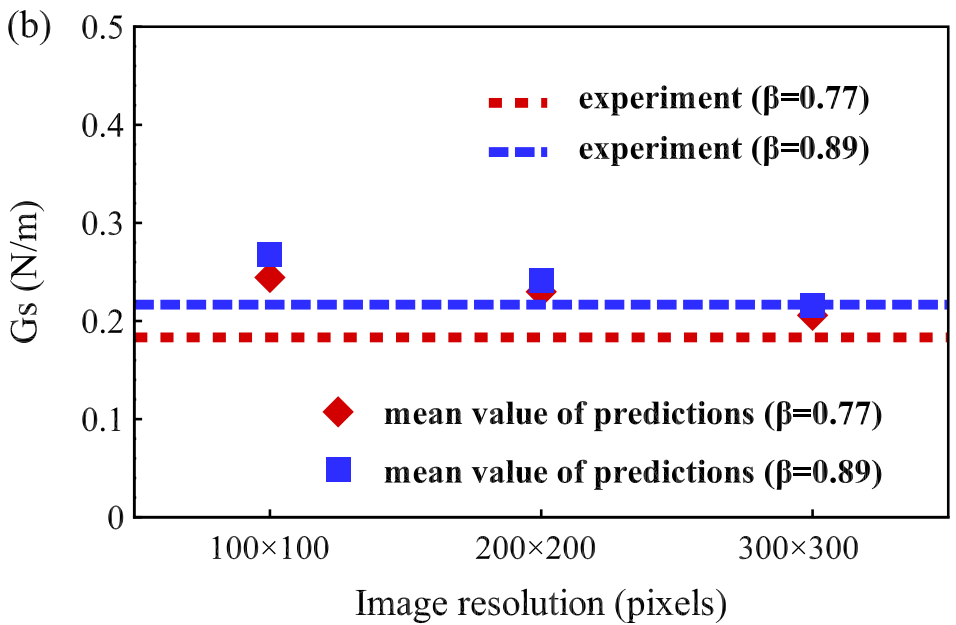

Figure 10: (a) Binary images of a deformed capsule with three levels of resolution: from left to right $100 \times 100$, $200 \times 200,300 \times 300$ pixels. The original image is taken from an experiment [39] at $\beta=0.77$ and $C a=0.052$. (b) Comparison of predicted membrane shear elasticity from images with different resolutions with experimental measurements [39].

value of 3\%. Both are consistent with a previous study use an inverse method [58]. The predicted membrane shear elasticity $G_{s}$ are presented in Fig. 9 and are compared with experiments. Reasonably good agreements can be observed for capsules with both sizes. Note that the experiments have measured the membrane area dilation elastic modulus $K_{s}$, which is related to $G_{s}$ by $K_{s}=3 G_{s}$. We find that the mean values of predicted $G_{s}$ only deviate from experimental measurements by $12 \%$ and $0.5 \%$, for capsules with $\beta=0.77$ and 0.89 , respectively. Note that in experiments the capsule profiles are not perfectly symmetric, which may have affected the accuracy of the present prediction. One example can be seen in Fig. 9(a) for a capsule with $\beta=0.77$ at $C a=0.052$.

In experiments capsule images may have different resolutions which will affect the performance of the present method. To test this, we consider capsule images with three levels of resolution (illustrated in Fig. 10(a)). Note that both training and testing samples, with various resolutions, are prepared using the same cases employed in Fig. 9. From Fig. 10(b), it is seen that with the image resolution dropping to 100 pixels in each direction, deviation of the predicted $G_{s}$ from the experimental measurement has increased 
significantly. However, the sacrifice of prediction accuracy is associated with a significant increase of prediction throughput rate, which has grown from 1400 to 3800 images per second.

\section{Conclusions}

We have developed a novel approach by integrating a DCNN with mechanistic capsule modelling to predict the mechanical properties of flowing capsules. The method considers the entire cross-sectional or footprint profile of the steady deformed capsule, and its accuracy has been demonstrated through tests against both numerical and experimental data. A significant merit of the approach is that unlike conventional inverse methods, which need to conduct a time-consuming online process to identify the best fit, the present DCNN is trained offline and its prediction process only involves a limited number of algebraic calculations. The present method can therefore increase the prediction throughput rate by a few orders of magnitude and process more than one thousand capsules per second. The method is flexible in that it can deal with both cross-sectional and footprint capsule profiles with comparable high accuracy. It can also characterise capsules with large deformation beyond Stokes flow regime, due to the versatility of the present mechanical models for both capsule dynamics and fluid flows. Furthermore, the method can predict multiple membrane parameters, such as the shear elasticity, area dilatation modulus and initial inflation, from a single steady capsule profile.

We have also attempted to uncover the mechanism that the DCNN makes decisions by considering its feature maps. The results suggest that the DCNN can successfully learn geometrical features of the capsule profile that are the most sensitive to the membrane law and its associated parameters. These features provide important information to the development of high-accuracy inverse methods which predict mechanical properties of flowing capsules from their steady deformed profiles.

Our new approach provides a promising tool which may enable high-throughput mechanistic characterisation of capsules and biological cells in microfluidic flows. However, for practical unknown capsules there may be a range of possibilities in membrane constitutive law and associated parameters. Biological cells usually have complicated internal structures such as the cytoskeleton and nucleus. In principle the present method can be extended to cover additional membrane constitutive laws, cell internal structures and a much larger parametric space. For the new purposes, the mechanical model, the DCNN architecture and its training samples will all need to be expanded.

\section{Acknowledgements}

This work was partially supported by the UK Engineering and Physical Science Research Council (EP/K000128/1) and the China Scholarship Council. 


\section{References}

[1] D. BARTHÈs-BieSel, Motion and deformation of elastic capsules and vesicles in flow, Annu. Rev. Fluid Mech., 48 (2016), pp. 25-52.

[2] M. Lorch, M. J. Thomasson, A. Diego-Taboada, S. Barrier, S. L. AtKin, G. MACKENZIE, AND S. J. ARCHIBALD, Mri contrast agent delivery using spore capsules: controlled release in blood plasma, Chem. Commun., 42 (2009), pp. 6442-6444.

[3] S. V. BhujBAL, P. De VOS, AND S. P. NiClOU, Drug and cell encapsulation: alternative delivery options for the treatment of malignant brain tumors, Adv. Drug Deliver. Rev., 67 (2014), pp. 142153.

[4] A. E. Mayfield, E. L. Tilokee, N. Latham, B. McNeill, B.-K. Lam, M. Ruel, E. J. SuUronen, D. W. Courtman, D. J. STEWART, AND D. R. DAVIS, The effect of encapsulation of cardiac stem cells within matrix-enriched hydrogel capsules on cell survival, post-ischemic cell retention and cardiac function, Biomaterials, 35(1) (2014), pp. 133-142.

[5] C. T. LIM, E. H. ZHOU, AND S. T. QUEK, Mechanical models for living cells-a review, J. Biomech., 39(2) (2006), pp. 195-216.

[6] A. MietKe, O. Otto, S. Girardo, P. Rosendahl, A. Taubenberger, S. Golfier, E. UlBRICHT, S. ALAND, J. GUCK, AND E. FISCHER-FRIEDRICH, Extracting cell stiffness from realtime deformability cytometry: theory and experiment, Biophys. J., 109(10) (2015), pp. 2023-2036.

[7] M. Laumann, W. Schmidt, A. Farutin, D. Kienle, S. Förster, C. Misbah, AND W. ZIMMERMANN, Emerging attractor in wavy poiseuille flows triggers sorting of biological cells, Phys. Rev. Lett., 122(12) (2019), 128002.

[8] D. R. Gossett, H. T. K. Tse, S. A. Lee, Y. Ying, A. G. Lindgren, O. O. Yang, J. RaO, A. T. CLARK, AND D. DI CARLO, Hydrodynamic stretching of single cells for large population mechanical phenotyping, Proc. Natl. Acad. Sci. U.S.A., 109(20) (2012), pp. 7630-7635.

[9] O. Otto, P. Rosendahl, A. Mietke, S. Golfier, C. Herold, D. Klaue, S. Girardo, S. PAGLIARA, A. EKPENYONG, A. JACOBI, ET AL., Real-time deformability cytometry: on-the-fly cell mechanical phenotyping, Nat. Methods, 12(3) (2015), 199.

[10] P. Rosendahl, K. Plak, A. Jacobi, M. Kraeter, N. Toepfner, O. Otto, C. Herold, M. WinZI, M. HerbIG, Y. GE, ET AL., Real-time fluorescence and deformability cytometry, Nat. Methods, 15(5) (2018), 355.

[11] M. RACHIK, D. BARTHÈs-BIESEL, M. CARIN, AND F. EDWARDS-LÉVY, Identification of a bioartificial microcapsule wall material parameter with an inverse method and the compression test, J. Colloid Interface Sci., 301 (2006), pp. 217-226.

[12] R. M. Hochmuth, Micropipette aspiration of living cells, J. Biomech., 33(1) (2000), pp. 15-22.

[13] J. GUCK, S. SCHINKINGER, B. LinCOLN, F. WOTTAWAH, S. EBERT, M. ROMEYKE, D. LENZ, H. M. ERICKSON, R. ANANTHAKRISHNAN, D. MitChell, et Al., Optical deformability as an inherent cell marker for testing malignant transformation and metastatic competence, Biophys. J., 88(5) (2005), pp. 689-698.

[14] Z. L. ZHOU, A. H. W. NGAN, B. TANG, AND A. X. WANG., Reliable measurement of elastic modulus of cells by nanoindentation in an atomic force microscope, J. Mech. Behav. Biomed., 8 (2012), pp. 134-142.

[15] K. S. CHANG AND W. L. OlbRICHT, Experimental studies of the deformation and breakup of a synthetic capsule in steady and unsteady simple shear flow, J. Fluid Mech., 250 (1993), pp. 609-633.

[16] A. WAlter, H. Rehage, AND H. LeONHARD, Shear induced deformation of microcapsules: shape oscillations and membrane folding, Colloids Surf. A Physicochem. Eng. Asp., 183 (2001), pp. 123-132. 
[17] G. Pieper, H. Rehage, AND D. BARTHÈs-Biesel, Deformation of a capsule in a spinning drop apparatus, J. Colloid Interface Sci., 202(2) (1998), pp. 293-300.

[18] M. Husmann, H. Rehage, E. Dhenin, AND D. BARTHÈs-Biesel, Deformation and bursting of nonspherical polysiloxane microcapsules in a spinning-drop apparatus, J. Colloid Interface Sci., 282(1) (2005), pp. 109-119.

[19] C. De Loubens, J. Deschamps, M. Georgelin, A. Charrier, F. Edwards-Lévy, AND M. LEONETTI, Mechanical characterization of cross-linked serum albumin microcapsules, Soft Matter, 10(25) (2014), pp. 4561-4568.

[20] C. De Loubens, J. Deschamps, G. Boedec, AND M. LeONetTI, Stretching of capsules in an elongation flow, a route to constitutive law, J. Fluid Mech., 767 (2015).

[21] E. M DARLING AND D. DI CARLO, High-throughput assessment of cellular mechanical properties, Annu. Rev. Biomed. Eng., 17 (2015), pp. 35-62.

[22] Y. LefebVRe, E. LeClerC, D. BARTHÈS-Biesel, J. WALter, AND F. EdWARdS-LÉvy, Flow of artificial microcapsules in microfluidic channels: a method for determining the elastic properties of the membrane, Phys. Fluids, 20(12) (2008), 123102.

[23] T. X. Chu, A.-V. SAlsaC, E. LeClerc, D. BARThÈs-Biesel, H. WurtZ, AND F. EdWARdSLÉVY, Comparison between measurements of elasticity and free amino group content of ovalbumin microcapsule membranes: discrimination of the cross-linking degree, J. Colloid Interface Sci., 355(1) (2011), pp. 81-88.

[24] X.-Q. Hu, B. SÉvéniÉ, A.-V. SAlsaC, E. LeClerC, AND D. BARTHÈs-Biesel, Characterizing the membrane properties of capsules flowing in a square-section microfluidic channel: Effects of the membrane constitutive law, Phys. Rev. E, 87(6) (2013), 063008.

[25] B. Fregin, F. CZerwinski, D. Biedenweg, S. Girardo, S. Gross, K. Aurich, And O. Отто, High-throughput single-cell rheology in complex samples by dynamic real-time deformability cytometry, Nat. Commun., 10(1) (2019), 415.

[26] F. J. Armistead, J. G. De Pablo, H. Gadêlha, S. A. Peyman, and S. D. Evans, Cells under stress: an inertial-shear microfluidic determination of cell behavior, Biophys. J., 116(6) (2019), pp. 1127-1135.

[27] Y. LeCun, Y. BengiO, AND G. Hinton, Deep learning, Nature, 521(7553) (2015), 436.

[28] M. T. MCCANN, K. H. JIN, AND M. UNSER, Convolutional neural networks for inverse problems in imaging: A review, IEEE Signal Process. Mag., 34(6) (2017), pp. 85-95.

[29] A. Kinm, L. KAestner, C. WAGner, AND S. QUint, Classification of red blood cell shapes in flow using outlier tolerant machine learning, PLoS Comput. Biol., 14(6) (2018), e1006278.

[30] V. SEKAR, M. ZHANG, C. SHU, AND B. C. KHOO, Inverse design of airfoil using a deep convolutional neural network, AIAA J., 57(3) (2019), pp. 993-1003.

[31] K. Duraisamy, G. IACCARINO, AND H. XiAO, Turbulence modeling in the age of data, Annu. Rev. Fluid Mech., 51 (2019), pp. 357-377.

[32] K. FukAmi, Y. NAbAe, K. KaWAi, AND K. FuKagata, Synthetic turbulent inflow generator using machine learning, Phys. Rev. Fluids, 4(6) (2019), 064603.

[33] J. KIM AND C. LEE, Prediction of turbulent heat transfer using convolutional neural networks, J. Fluid Mech., 882 (2020), 18.

[34] A. E. GReEn AND J. E. AdKINS, Large Elastic Deformations and Non-Linear Continuum Mechanics, Clarendon Press, Oxford, UK, 1960.

[35] R. SKalaK, A. TOZEREN, R. P. ZARDA, AND S. CHIEN, Strain energy function of red blood cell membranes, Biophys. J., 13(3) (1973), pp. 245-264.

[36] O.-Y. ZHONG-CAN AND W. HELFRICH, Bending energy of vesicle membranes: general expressions for the first, second, and third variation of the shape energy and applications to spheres and 
cylinders, Phys. Rev. A, 39(10) (1989), 5280.

[37] D. CORDASCO AND P. BAGCHI, Orbital drift of capsules and red blood cells in shear flow, Phys. Fluids, 25(9) (2013), 091902.

[38] C. Dupont, A.-V. Salsac, D. Barthès-Biesel, M. Vidrascu, and P. Le Tallec, Influence of bending resistance on the dynamics of a spherical capsule in shear flow, Phys. Fluids, 27 (2015), 051902.

[39] F. Risso, F. Collé-Paillot, AND M. Zagzoule, Experimental investigation of a bioartificial capsule flowing in a narrow tube, J. Fluid Mech., 547 (2006), pp. 149-173.

[40] Y. SUI, Y. T. CHEW, P. ROY, AND H. T. LOW, A hybrid method to study flow-induced deformation of three-dimensional capsules, J. Comput. Phys., 227(12) (2008), pp. 6351-6371.

[41] Y. SuI, H. T. LOW, Y. T. CHEW, AND P. ROY, Tank-treading, swinging, and tumbling of liquidfilled elastic capsules in shear flow, Phys. Rev. E, 77 (2008), 016310.

[42] Y. SuI, Y. T. CHEW, P. RoY, Y. P. CHENG, AND H. T. LOW, Dynamic motion of red blood cells in simple shear flow, Phys. Fluids, 20(11) (2008), 112106.

[43] Y. SUI, H. T. LOW, Y. T. CHEW, AND P. ROY, A front-tracking lattice boltzmann method to study flow-induced deformation of three-dimensional capsules, Comput. Fluids, 39(3) (2010), pp. 499-511.

[44] Z. WANG, Y. SUI, P. D. M. SPELT, AND W. WANG, Three-dimensional dynamics of oblate and prolate capsules in shear flow, Phys. Rev. E, 88(5) (2013), 053021.

[45] Z. WANG, Y. Sui, A.-V. SAlsaC, D. BARTHÈs-BiESel, AND W. WANG, Motion of a spherical capsule in branched tube flow with finite inertia, J. Fluid Mech., 806 (2016), pp. 603-626.

[46] Z. WANG, Y. SUI, A.-V. SALSAC, D. BARTHÈs-BIESEL, AND W. WANG, Path selection of a spherical capsule in a microfluidic branched channel: towards the design of an enrichment device, J. Fluid Mech., 849 (2018), pp. 136-162.

[47] M. Abadi, P. Barham, J. Chen, Z. Chen, A. Davis, J. Dean, M. Devin, S. Ghemawat, G. IRVING, M. ISARD, ET AL., Tensorflow: A system for large-scale machine learning, in Proceedings of 12th USENIX Symposium on Operating Systems Design and Implementation, pages 265-283, California, USA, 2016. USENIX Association.

[48] A. Gulli AND S. PAL, Deep Learning with Keras, Packt Publishing Ltd, Birmingham, UK, 2017.

[49] G. E. DAhL, T. N. SAINATH, AND G. E. HintON, Improving deep neural networks for locsr using rectified linear units and dropout, in Proceedings of 38th International Conference on Acoustics, Speech and Signal Processing, pages 8609-8613, Vancouver, Canada, 2013. IEEE.

[50] R. RubinsteIN, The cross-entropy method for combinatorial and continuous optimization, Methodol. Comput. Appl. Probab., 1(2) (1999), pp. 127-190.

[51] D. E. Rumelhart, G. E. Hinton, AND R. J. Williams, Learning representations by backpropagating errors, Nature, 323(6088) (1986), pp. 533-536.

[52] D. P. Kingma AND J. L. BA, Adam: A method for stochastic optimization, arXiv preprint arXiv:1412.6980, 2014.

[53] S. IOFFE AND C. SZEGEDY, Batch normalization: accelerating deep network training by reducing internal covariate shift, arXiv preprint arXiv:1502.03167, 2015.

[54] N. SRIVASTAVA, Improving Neural Networks with Dropout, Master thesis, University of Toronto, 2013.

[55] D. BARTHÈS-BIESEL, A. DiAZ, AND E. DHENIN, Effect of constitutive laws for two-dimensional membranes on flow-induced capsule deformation, J. Fluid Mech., 460:211, 2002.

[56] M. P. Dubuisson AND A. K. JAIN, A modified hausdorff distance for object matching, in Proceedings of 12 th international conference on pattern recognition, pages 566-568, Jerusalem, 
Israel, 1994. IEEE.

[57] F. RISSO AND M. CARIN, Compression of a capsule: mechanical laws of membranes with negligible bending stiffness, Phys. Rev. E, 69(6) (2004), 061601.

[58] Y. LEFEBVRE AND D. BARTHÈS-BIESEL, Motion of a capsule in a cylindrical tube: effect of membrane pre-stress, J. Fluid Mech., 589 (2007), pp. 157-181. 